\title{
Kernos
}

Revue internationale et pluridisciplinaire de religion grecque antique

31 | 2018

Varia

\section{Animal Sacrifice in Ancient Greece}

\section{Zoé Pitz}

\section{OpenEdition}

Journals

\section{Édition électronique}

URL : http://journals.openedition.org/kernos/2840

DOI : 10.4000/kernos.2840

ISSN : 2034-7871

\section{Éditeur}

Centre international d'étude de la religion grecque antique

\section{Édition imprimée}

Date de publication : 1 décembre 2018

Pagination : 317-318

ISBN : 978-2-87562-055-2

ISSN : 0776-3824

\section{Référence électronique}

Zoé Pitz, "Animal Sacrifice in Ancient Greece », Kernos [En ligne], 31 | 2018, mis en ligne le 01 octobre 2018, consulté le 25 janvier 2021. URL : http://journals.openedition.org/kernos/2840 ; DOI : https:// doi.org/10.4000/kernos.2840

Ce document a été généré automatiquement le 25 janvier 2021.

Kernos 


\title{
Animal Sacrifice in Ancient Greece
}

\author{
Zoé Pitz
}

\section{RÉFÉRENCE}

Krzysztof BIELAWSKI (éd.), Animal Sacrifice in Ancient Greece. Proceedings of the First International Workshops in Kraków (12-14.11.2015), Warszawa, Wydawnictwo Naukowe Sub Lupa, 2017. 1 vol. $17 \times 24 \mathrm{~cm}, 227$ p. ISBN : 978-83-65886-02-6.

1 Cet ouvrage rassemble les actes du premier séminaire international de Cracovie sur le sacrifice animal en Grèce ancienne. Il s'ouvre sur une introduction de R. Parker et compte neuf articles. - S. Scullion s'interroge sur les sacrifices animaux de " pénalité ", imposés suite à la violation de règles cultuelles. De tels sacrifices, qui fonctionnent comme des compléments obligatoires à des sacrifices purificatoires, sont attestés à plusieurs reprises dans l'inscription de Marmarini publiée en 2015 ainsi que dans la "loi cathartique» de Cyrène. L'A. analyse en particulier le langage employé pour désigner ces sacrifices de "pénalité ». - Le concept de $\delta \varepsilon \imath \sigma l \delta \alpha \imath \mu o v i ́ \alpha$ (littéralement " peur des dieux ») est étudié par W. Lengauer. Selon l'A., ce concept se trouve à la base

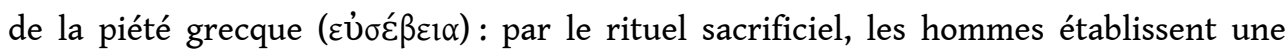
forme de partage avec les divinités et leur prouvent qu'ils font preuve de respect envers elles. De cette manière, ils tentent de surmonter leur peur des dieux et de s'assurer que ces derniers ne se sentiront pas offensés. - G. Ekroth s'interroge sur la pratique de l'holocauste dans la religion grecque ancienne et sur la pertinence de considérer ce type de sacrifice comme le rituel générique accompli dans les cultes de divinités chthoniennes. Selon l'A., toutes les sources suggèrent que l'holocauste n'occupait pas une place importante dans la pratique rituelle, et la rareté de ces sacrifices serait plus simple à expliquer si on les considère comme des powerful actions ou heilige Handlungen - employées pour compléter, modifier ou remplacer la thysia que si on les interprète comme des rituels «standards " accomplis pour les divinités chthoniennes (modèle théorique "olympien-chthonien»). - V.Pirenne-Delforge revient sur une question qui a déjà fait l'objet de nombreuses discussions depuis la publication de la tablette rituelle de Sélinonte, celle de l'identité complexe des 
Tritopatores. Au lieu de considérer, à l'instar de K. Clinton - dont l'hypothèse a été largement adoptée jusqu'ici -, qu'il existait deux groupes différents d'ancêtres, les uns impurs et les autres purs, l'A. suggère de voir les Tritopatores purs et impurs comme un seul et même groupe d'ancêtres. - Une analyse comparative du sacrifice grec et du sacrifice védique est proposée par R. Seaford. Après avoir décrit certaines différences fondamentales entre les deux pratiques, l'A. tente de relier ces différences, de même que la pensée "philosophique» naissante des deux cultures, aux développements socio-économiques observés entre 700 et 400 av. J.-C., en particulier la monétisation. L'article de K. Bielawski fournit un commentaire détaillé de toutes les références à des sacrifices animaux présentes dans les "inscriptions éleusiennes", c'est-à-dire les inscriptions associées aux sanctuaires d'Éleusis, non seulement au sens topographique, mais également en terme de contenu. Les inscriptions concernées par un commentaire sont les suivantes : IG $\mathrm{I}^{2} 5$, IG $\mathrm{I}^{3} 32, I G \mathrm{I}^{3} 78 \mathrm{a}$, IG $\mathrm{II}^{2} 1672$ et $I G \mathrm{II}^{2}$ 1673. - B. Bednarek étudie la signification du terme oủ $\lambda \alpha$ é et son usage chez Homère, ainsi que son rapport

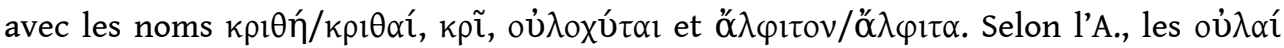
étaient probablement des grains d'orge non moulus mélangés avec du sel, qui étaient distribués à ceux qui prenaient part au sacrifice et qui étaient jetés sur l'animal sacrificiel et l'autel durant ou immédiatement après la prière. $-P$. Biernat analyse le rituel de serment accompli par les dix rois d'Atlantis, décrit dans le Critias de Platon. L'A. tente de mettre en lumière les éléments inspirés de la pratique sacrificielle grecque réelle et s'intéresse à l'intertextualité de ce rituel, sur lequel les chercheurs se sont généralement peu penchés jusqu'à présent, sans doute en raison de son caractère fictif. - Enfin, L.Trzcionkowski aborde le problème de la tradition et de l'innovation dans le rituel sacrificiel grec via l'analyse de la terminologie. L'A. étudie l'usage des trois termes techniques attestés dans le langage épique pour désigner les fémurs: $\mu \tilde{\eta} \rho \alpha$, $\mu \eta \rho i ́ \alpha$ et $\mu \eta \rho o i ́$. La distribution de ces mots dans l'épopée homérique suggèrerait qu'ils ne doivent pas être considérés comme de purs synonymes.

\section{AUTEURS}

\section{ZOÉ PITZ}

F.R.S.-FNRS - Université de Liège 